\title{
Impact of Resting Heart Rate on Cardiovascular Mortality According to Serum Albumin Levels in a 24-year Follow-up Study on a General Japanese Population: NIPPON DATA80
}

\author{
Yiwei Liu ${ }^{1}$, Aya Hirata ${ }^{1}$, Tomonori Okamura ${ }^{1}$, Daisuke Sugiyama ${ }^{2}$, Takumi Hirata ${ }^{3}$, Aya Kadota ${ }^{4}$, \\ Keiko Kondo ${ }^{4}$, Takayoshi Ohkubo ${ }^{5}$, Katsuyuki Miura ${ }^{4,6}$, Akira Okayama ${ }^{7}$, and Hirotsugu Ueshima ${ }^{4,6}$, \\ for the NIPPON DATA80 Research Group
}

\author{
${ }^{1}$ Department of Preventive Medicine and Public Health, Keio University School of Medicine, Tokyo, Japan \\ ${ }^{2}$ Faculty of Nursing and Medical Care, Keio University, Fujisawa, Kanagawa, Japan \\ ${ }^{3}$ Department of Public Health, Hokkaido University Faculty of Medicine, Sapporo, Japan \\ ${ }^{4}$ Department of Public Health, Shiga University of Medical Science, Shiga, Japan \\ ${ }^{5}$ Department of Hygiene and Public Health, Teikyo University School of Medicine, Tokyo, Japan \\ ${ }^{6}$ Center for Epidemiologic Research in Asia, Shiga University of Medical Science, Shiga, Japan \\ ${ }^{7}$ Research Institute of Strategy for Prevention, Tokyo, Japan
}

Received March 22, 2021; accepted August 26, 2021; released online September 11, 2021

\begin{abstract}
Background: Elevated resting heart rate (RHR) is associated with an increased risk of cardiovascular disease (CVD) and allcause mortality. However, the findings of cohort studies differed. Thus, the impact of RHR on CVD mortality might be different according to the background of the population. Therefore, we examined the relationship of RHR and CVD mortality according to serum albumin (ALB) levels in a Japanese general population.

Methods: In total, 8,363 individuals without a history of CVD were followed for 24.0 years. The participants were divided into four groups according to the quartiles of RHR (Q1-Q4), and they were further classified into the high and low ALB groups based on a median value of $44 \mathrm{~g} / \mathrm{L}$. We estimated the multivariable-adjusted hazard ratios (HRs) of CVD mortality in each RHR group based on ALB levels, and the interaction between RHR and ALB groups on CVD mortality was evaluated.

Results: We found no significant association between RHR and CVD mortality. However, the Q4 of RHR was significantly associated with an increased risk for CVD mortality (HR 1.27; 95\% confidence interval [CI], 1.02-1.57) in participants with a low ALB level. Meanwhile, the Q4 of RHR was significantly correlated with a decreased risk for CVD morality in those with a high ALB level (HR 0.61; 95\% CI, 0.47-0.79) after adjusting for covariates. A significant interaction between RHR and ALB for CVD mortality was shown $(P<0.001)$.

Conclusions: The impact of RHR on CVD mortality differed according to ALB levels in a general Japanese population.
\end{abstract}

Key words: cardiovascular disease; mortality; serum albumin; resting heart rate; cohort study

Copyright (C) 2021 Yiwei Liu et al. This is an open access article distributed under the terms of Creative Commons Attribution License, which permits unrestricted use, distribution, and reproduction in any medium, provided the original author and source are credited.

\section{INTRODUCTION}

Elevated resting heart rate (RHR) is associated with an increased risk of coronary heart disease $(\mathrm{CHD})^{1,2}$ heart failure $\left.(\mathrm{HF})\right)^{3,4}$ cardiovascular disease (CVD), ${ }^{5,6}$ and all-cause mortality ${ }^{7,8}$ in the general population. This association is observed in all age groups in different clinical settings, irrespective of other atherosclerotic risk factors. ${ }^{9,10}$ However, the mechanism underlying the relationship between RHR and cause-specific mortality or morbidity remains unknown. To date, clinical trials have not shown the importance of slow RHR in hypertensive patients without CVD. ${ }^{10,11}$ Accordingly, comorbidities underlying a high RHR should be further validated. A high RHR is an index of increased sympathetic nervous system activity, ${ }^{12}$ which might be associated with the development of arrythmia, arterial stiffness, and hypertension. In the general population, sympathetic nervous system activity is commonly accelerated by individual responses to continuous mental stress. ${ }^{13}$ Further, lack of regular aerobic exercise, smoking, and heavy alcohol drinking are associated with a high RHR. ${ }^{14}$

Serum albumin (ALB) is a nutritional factor influencing general health status. ${ }^{15}$ Moreover, it has anti-inflammatory, antioxidant, anticoagulant, and antiplatelet aggregation properties. From a mechanism perspective, ALB is able to combine with nitric oxide $(\mathrm{NO})^{16}$ to maintain a normal level. ALB is also involved in recovery from ischemia injury. ${ }^{17} \mathrm{ALB}$, even within the clinical normal range, can significantly influence the incidence of CVD and all-cause mortality. ${ }^{18}$ Such subclinical 
inflammation might increase RHR and decrease heart-rate variability, ultimately leading to mortality. ${ }^{19}$ A previous study has shown that nutritional intake influences RHR variability. ${ }^{20}$ However, to the best of our knowledge, the association of ALB level and RHR with mortality has not been comprehensively assessed. To validate this notion, we conducted a long-term cohort study to assess the impact of RHR on cause-specific mortality according to ALB levels.

\section{METHODS}

\section{Study participants}

We performed a cohort study based on the National Survey on Circulatory Disorders in 1980, which was referred to as the National Integrated Project for Prospective Observation of Non-communicable Diseases and Its Trends in the Aged, 1980 (NIPPON DATA 80). Details of the cohort are provided elsewhere. ${ }^{21-23}$ In brief, we randomly selected 300 districts in Japan, and a total of 10,546 participants (4,640 men and 5,906 women) aged $>30$ years participated in the baseline survey, with a participation rate of $77 \%$. Details of baseline examinations and follow-up surveys are provided in eMaterials 1 .

Of 10,546 participants, 2,183 were excluded due to the following reasons: history of CVD $(n=676)$, missing information at baseline survey $(n=598)$, and lost to follow-up because of incomplete access to residential information $(n=909)$. There was no selection bias in terms of baseline characteristics between participants with and without follow-up. Finally, 8,363 participants (3,666 men and 4,697 women) were included in the analysis.

\section{Ethical approval}

The current study was conducted in accordance with the ethical guidelines of Shiga University of Medical Science (R2005-021) and Keio University School of Medicine (2018-0108).

\section{Statistical analysis}

The participants were divided into four groups according to RHR (beats/min) quartiles (Q1-Q4) as follows: Q1, <62; Q2, 62-68; Q3, 69-77; and Q4, >77 beats/min in all participants; Q1, <60; Q2, 60-66; Q3, 67-73; and Q4, >73 beats in men; and Q1, <64; Q2, 64-70; Q3, 71-77; and Q4, >77 beats in women. The mean values and standard deviation were presented as continuous variables, and the number and proportion as categorical variables according to each RHR category. Continuous variables were compared between groups using one-way analysis of variance, and categorical variables were compared using the $\chi 2$-test. We assessed the age- and multivariable-adjusted hazard ratios (HRs) and $95 \%$ confidence intervals (CIs) of each RHR category for allcause death, total CVD death, cause-specific CVD mortality, and non-CVD mortality using a Cox proportional hazards model. Model 1 was adjusted for age; model 2 for the variable in model 1 plus body mass index, blood glucose levels, systolic blood pressure, hypertension treatment, total cholesterol level, smoking status, and alcohol drinking status; and model 3 for the variables in model 2 plus ALB level. Then, sex was adjusted in the sexcombined analysis. Further, we estimated the HRs of RHR quartiles according to ALB levels based on the median values (low: $<44$ and high: $\geq 44 \mathrm{~g} / \mathrm{L}$ ) for outcomes using models 1 and 2. In addition, we generated the interaction term by multiplying RHR as a continuous variable and ALB groups, and evaluated the interaction on all-cause, CVD, and non-CVD mortality in model 2 using a Cox proportional hazards. To validate the influence of arrhythmia on HR, we performed the same analysis with exclusion of individuals with atrial fibrillation or frequent supraventricular and/or ventricular premature beats. Two-sided $P$-values of $<0.05$ were considered statistically significant. For baseline characteristics, we used one-way ANOVA to test if covariates were distributed equally between groups. Statistical analysis was performed with the $\mathrm{R}$ package version 3.6.1 (R Foundation for Statistical Computing, Vienna, Austria).

\section{RESULTS}

The characteristics of participants according to RHR quantiles at the baseline survey are shown in Table 1 . The mean non-fasting blood glucose levels were high in both men and women with a high RHR. The proportion of individuals receiving hypertension treatment was highest among women in the group with the lowest RHR.

During a mean follow-up of 24.0 years, with 203,021 personyears of observation time, the total number of deaths was 3,128 (1,055 CVD deaths and 2,073 non-CVD deaths). Among CVD deaths, 219 were caused by CHD and 455 by stroke. The crude mortality rates and $\mathrm{HRs}$ for all-cause, CVD, and non-CVD mortality according to RHR quartiles are presented in Table 2. Compared with Q1, Q4 was significantly associated with an increased risk for all-cause mortality after adjusting for confounders in all participants (HR 1.15; 95\% CI, 1.04-1.28). In addition, Q4 was significantly associated with an increased risk for non-CVD mortality in both men and women (HR 1.21; 95\% CI, $1.03-1.41$ vs HR 1.19; 95\% CI, 1.00-1.43). Meanwhile, RHR was not significantly associated with an increased risk for CVD mortality. ALB level was significantly associated with CVD mortality (HR 0.49; 95\% CI, 0.38-0.65).

The crude mortality rates and HRs for all-cause, CVD, and non-CVD mortality according to RHR quartiles in the low and high ALB level groups are depicted in Table 3. In the low ALB level group, compared with Q1, Q4 was significantly associated with an increased risk for all-cause mortality after adjusting for confounders in all participants and women (HR 1.35; 95\% CI, $1.18-1.53$ vs HR 1.24; 95\% CI, 1.03-1.49). Moreover, a correlation was observed between Q4 and an increased risk for CVD mortality in the low ALB level group (HR 1.27; 95\% CI, 1.02-1.57). Meanwhile, Q4 was significantly associated with a decreased risk for CVD morality in all participants in the high ALB level group (HR 0.61; 95\% CI, 0.47-0.79). Moreover, Q4 was significantly correlated with an increased risk for non-CVD mortality in all participants in the low ALB level group (HR 1.27; 95\% CI, 1.07-1.50). A significant interaction between RHR and ALB for CVD mortality was shown $(P<0.001)$; however, such an interaction was not evident for non-CVD mortality $(P=0.31)$. After excluding individuals with atrial fibrillation $(n=57)$ and frequent supraventricular and/or ventricular premature beats $(n=99)$, or those who received antihypertensive treatment, the results remained did not change significantly (data not shown).

\section{DISCUSSION}

The current study found no significant association between RHR and CVD mortality. In the analysis stratified according to ALB levels, a higher RHR was significantly associated with an 
Liu Y, et al.

Table 1. Characteristics of study participants according to resting heart rate quartiles at baseline survey in 1980

\begin{tabular}{|c|c|c|c|c|c|}
\hline Total (Quantiles in beats/min) & Q1 $(<62)$ & Q2 (62-68) & Q3 (69-77) & $\mathrm{Q} 4(>77)$ & $P$ value \\
\hline$N$ & 2,225 & 2,049 & 2,138 & 1,951 & - \\
\hline Age, years & $51(13)$ & 49 (13) & $49(13)$ & $50(14)$ & $<0.001$ \\
\hline Sex, male, $\%$ & 59 & 44 & 37 & 34 & $<0.001$ \\
\hline Smoking, \% & 33 & 29 & 26 & 26 & $<0.001$ \\
\hline Drinking, $\%$ & 39 & 38 & 35 & 32 & $<0.001$ \\
\hline Body mass index, $\mathrm{kg} / \mathrm{m}^{2}$ & $22(2.9)$ & $23(3.1)$ & $23(3.5)$ & $23(3.5)$ & 0.01 \\
\hline Total cholesterol, mmol/L & $4.7(0.8)$ & $4.7(0.9)$ & $4.7(0.9)$ & $4.8(0.8)$ & $<0.001$ \\
\hline Non-fasting blood glucose, $\mathrm{mmol} / \mathrm{L}$ & $6.9(1.6)$ & $7.1(1.9)$ & $7.2(1.8)$ & $7.7(2.4)$ & $<0.001$ \\
\hline Systolic blood pressure, $\mathrm{mm} \mathrm{Hg}$ & $138(23)$ & $135(22)$ & $136(23)$ & $139(22)$ & $<0.001$ \\
\hline Serum albumin, $\mathrm{g} / \mathrm{L}$ & $44(2.6)$ & $44(2.6)$ & $44(2.6)$ & $44(2.8)$ & 0.04 \\
\hline Hypertension treatment, $\%$ & 15.1 & 14 & 11 & 9.6 & 0.09 \\
\hline Men (Quantiles in beats/min) & Q1 $(<60)$ & Q2 (60-66) & Q3 (67-73) & $\mathrm{Q} 4(>73)$ & $P$ value \\
\hline$N$ & 903 & 910 & 945 & 908 & - \\
\hline Age, years & $51(13)$ & $50(13)$ & 49 (13) & $50(13)$ & 0.08 \\
\hline Smoking, \% & 56.3 & 63.3 & 64.9 & 67.1 & $<0.001$ \\
\hline Drinking, $\%$ & 73.3 & 75.1 & 74.1 & 75.8 & 0.82 \\
\hline Body mass index, $\mathrm{kg} / \mathrm{m}^{2}$ & $22(2.8)$ & $22(2.7)$ & $23(2.8)$ & $23(3.1)$ & $<0.001$ \\
\hline Total cholesterol, mmol/L & $4.8(0.8)$ & $4.8(0.9)$ & $4.9(0.8)$ & $5.0(0.9)$ & $<0.001$ \\
\hline Non-fasting blood glucose, $\mathrm{mmol} / \mathrm{L}$ & $6.9(1.7)$ & $7.1(2.0)$ & $7.3(1.8)$ & $7.8(2.6)$ & $<0.001$ \\
\hline Systolic blood pressure, $\mathrm{mm} \mathrm{Hg}$ & $135(21)$ & $137(20)$ & $139(21)$ & $143(21)$ & $<0.001$ \\
\hline Serum albumin, $\mathrm{g} / \mathrm{L}$ & $44(3.1)$ & $44(3.0)$ & $45(3.0)$ & $44(3.0)$ & 0.002 \\
\hline Hypertension treatment, \% & 9.2 & 10.8 & 8.5 & 10.9 & 0.19 \\
\hline Women (Quantiles in beats/min) & Q1 $(<64)$ & Q2 (64-70) & Q3 (71-77) & $\mathrm{Q} 4(>77)$ & $P$ value \\
\hline$N$ & 1,273 & 1,151 & 1,102 & 1,171 & - \\
\hline Age, years & $52(13)$ & $50(13)$ & $50(14)$ & $50(14)$ & $<0.001$ \\
\hline Smoking, \% & 7.3 & 8.1 & 10.2 & 9.2 & 0.21 \\
\hline Drinking, $\%$ & 20.9 & 20.8 & 20.5 & 17.7 & 0.33 \\
\hline Body mass index, $\mathrm{kg} / \mathrm{m}^{2}$ & $23(3.0)$ & $23(3.4)$ & $23(3.5)$ & $23(3.6)$ & 0.87 \\
\hline Total cholesterol, mmol/L & $4.8(0.9)$ & $4.9(0.8)$ & $4.9(0.9)$ & $5.0(1.0)$ & 0.49 \\
\hline Non-fasting blood glucose, $\mathrm{mmol} / \mathrm{L}$ & $6.9(1.5)$ & $7.1(1.6)$ & $7.2(1.9)$ & $7.6(2.3)$ & $<0.001$ \\
\hline Systolic blood pressure, $\mathrm{mm} \mathrm{Hg}$ & $132(22)$ & $131(21)$ & $134(21)$ & $137(21)$ & $<0.001$ \\
\hline Serum albumin, $\mathrm{g} / \mathrm{L}$ & $43(2.2)$ & $44(2.3)$ & $44(2.9)$ & $44(2.9)$ & $<0.001$ \\
\hline Hypertension treatment, \% & 14.7 & 10.2 & 10.3 & 9.9 & $<0.001$ \\
\hline
\end{tabular}

Values reported as mean (standard deviation) unless otherwise noted.

Table 2. Crude mortality rates and hazard ratios for all-cause, cardiovascular disease (CVD) and non-CVD mortality according to resting heart rate $(\mathrm{RHR})$ quartiles

All-cause mortality

\begin{tabular}{|c|c|c|c|c|c|}
\hline Total & Quantiles (beats/min) & Q1 $(<62)$ & Q2 (62-68) & Q3 (69-77) & Q4 (>77) \\
\hline & Number of participants & 2,225 & 2,049 & 2,138 & 1,951 \\
\hline & Person-years & 53,526 & 51,022 & 52,743 & 46,836 \\
\hline & Number of events & 892 & 733 & 750 & 753 \\
\hline & Crude mortality & 16.6 & 14.4 & 14.2 & 16.1 \\
\hline & Hazard ratio & & & & \\
\hline & Model 1 & 1 & $1.07[0.97,1.18]$ & $1.07[0.97,1.19]$ & $1.21[1.10,1.34]^{* * *}$ \\
\hline & Model 2 & 1 & $1.03[0.94,1.14]$ & $1.03[0.93,1.14]$ & $1.11[1.00,1.23]^{*}$ \\
\hline & Model 3 & 1 & $1.04[0.94,1.15]$ & $1.04[0.95,1.15]$ & $1.15[1.04,1.28]^{*}$ \\
\hline \multirow[t]{9}{*}{ Men } & Quantiles (beats/min) & Q1 $(<60)$ & Q2 (60-66) & Q3 (67-73) & Q4 (>73) \\
\hline & Number of participants & 1,044 & 920 & 794 & 908 \\
\hline & Person-years & 24,376 & 21,914 & 19,057 & 20,579 \\
\hline & Number of events & 591 & 375 & 327 & 338 \\
\hline & Crude mortality & 24.2 & 17.1 & 17.1 & 16.4 \\
\hline & Hazard ratio & & & & \\
\hline & Model 1 & 1 & $1.05[0.92,1.21]$ & $0.99[0.86,1.14]$ & $1.21[1.06,1.37]^{* *}$ \\
\hline & Model 2 & 1 & $0.98[0.86,1.12]$ & $0.95[0.82,1.09]$ & $1.10[0.96,1.25]$ \\
\hline & Model 3 & 1 & $1.01[0.88,1.15]$ & $0.96[0.83,1.10]$ & $1.13[0.99,1.29]$ \\
\hline \multirow[t]{9}{*}{ Women } & Quantiles (beats/min) & Q1 $(<64)$ & Q2 (64-70) & Q3 (71-77) & Q4 (>77) \\
\hline & Number of participants & 1,273 & 1,151 & 1,102 & 1,171 \\
\hline & Person-years & 32,002 & 29,402 & 27,637 & 29,159 \\
\hline & Number of events & 414 & 346 & 357 & 380 \\
\hline & Crude mortality & 12.9 & 11.7 & 12.9 & 13.0 \\
\hline & Hazard ratio & & & & \\
\hline & Model 1 & 1 & $1.05[0.90,1.21]$ & $1.08[0.94,1.24]$ & $1.16[1.01,1.34]^{*}$ \\
\hline & Model 2 & 1 & $1.03[0.89,1.19]$ & $1.05[0.91,1.21]$ & $1.09[0.94,1.26]$ \\
\hline & Model 3 & 1 & $1.05[0.91,1.21]$ & $1.09[0.95,1.26]$ & $1.15[0.99,1.33]$ \\
\hline
\end{tabular}




\begin{tabular}{|c|c|c|c|c|c|}
\hline \multicolumn{6}{|c|}{ CVD mortality } \\
\hline \multirow[t]{9}{*}{ Total } & Quantiles (beats/min) & Q1 $(<62)$ & Q2 (62-68) & Q3 (69-77) & Q4 (>77) \\
\hline & Number of participants & 2,225 & 2,049 & 2,138 & 1,951 \\
\hline & Person-years & 53,526 & 51,022 & 52,743 & 46,836 \\
\hline & Number of events & 315 & 230 & 260 & 250 \\
\hline & Crude mortality & 5.9 & 4.5 & 4.9 & 5.3 \\
\hline & Hazard ratio & & & & \\
\hline & Model 1 & 1 & $0.93[0.78,1.10]$ & $1.02[0.86,1.20]$ & $1.07[0.91,1.27]$ \\
\hline & Model 2 & 1 & $0.87[0.74,1.04]$ & $0.94[0.80,1.11]$ & $0.93[0.78,1.10]$ \\
\hline & Model 3 & 1 & $0.88[0.74,1.05]$ & $0.96[0.81,1.14]$ & $0.98[0.82,1.16]$ \\
\hline \multirow[t]{9}{*}{ Men } & Quantiles (beats/min) & Q1 $(<60)$ & Q2 (60-66) & Q3 (67-73) & $\mathrm{Q} 4(>73)$ \\
\hline & Number of participants & 1,044 & 920 & 794 & 908 \\
\hline & Person-years & 24,376 & 21,914 & 19,057 & 20,579 \\
\hline & Number of events & 149 & 124 & 99 & 132 \\
\hline & Crude mortality & 6.1 & 5.7 & 5.2 & 6.4 \\
\hline & Hazard ratio & & & & \\
\hline & Model 1 & 1 & $1.07[0.84 .1 .36]$ & $0.99[0.77,1.28]$ & $1.14[0.90,1.45]$ \\
\hline & Model 2 & 1 & $0.96[0.76,1.22]$ & $0.90[0.70,1.17]$ & $0.96[0.75,1.22]$ \\
\hline & Model 3 & 1 & $0.98[0.77,1.25]$ & $0.92[0.71,1.18]$ & $0.99[0.78,1.26]$ \\
\hline \multirow[t]{9}{*}{ Women } & Quantiles (beats/min) & Q1 $(<64)$ & Q2 (64-70) & Q3 (71-77) & Q4 (>77) \\
\hline & Number of participants & 1,273 & 1,151 & 1,102 & 1,171 \\
\hline & Person-years & 32,002 & 29,402 & 27,637 & 29,159 \\
\hline & Number of events & 158 & 123 & 131 & 139 \\
\hline & Crude mortality & 4.9 & 4.2 & 4.7 & 4.8 \\
\hline & Hazard ratio & & & & \\
\hline & Model 1 & 1 & $0.98[0.77,1.24]$ & $1.01[0.80,1.27]$ & $1.08[0.86,1.36]$ \\
\hline & Model 2 & 1 & $0.97[0.76,1.23]$ & $0.99[0.78,1.26]$ & $0.99[0.78,1.26]$ \\
\hline & Model 3 & 1 & $0.99[0.78,1.26]$ & $1.05[0.83,1.33]$ & $1.08[0.85,1.37]$ \\
\hline \multicolumn{6}{|c|}{ Non-CVD mortality } \\
\hline \multirow[t]{9}{*}{ Total } & Quantiles (beats/min) & $\mathrm{Q} 1(<62)$ & Q2 (62-68) & Q3 (69-77) & $\mathrm{Q} 4(>77)$ \\
\hline & Number of participants & 2,225 & 2,049 & 2,138 & 1,951 \\
\hline & Person-years & 53,526 & 51,022 & 52,743 & 46,836 \\
\hline & Number of events & 577 & 503 & 490 & 503 \\
\hline & Crude mortality & 10.8 & 9.9 & 9.3 & 10.7 \\
\hline & Hazard ratio & & & & \\
\hline & Model 1 & 1 & $1.15[1.02,1.29]^{*}$ & $1.10[0.98,1.25]$ & $1.28[1.14,1.45]^{* * *}$ \\
\hline & Model 2 & 1 & $1.12[0.99,1.27]$ & $1.07[0.95,1.21]$ & $1.22[1.08,1.38]^{* *}$ \\
\hline & Model 3 & 1 & $1.13[1.01,1.28]^{*}$ & $1.09[0.96,1.23]$ & $1.26[1.11,1.42]^{* * *}$ \\
\hline \multirow[t]{9}{*}{ Men } & Quantiles (beats/min) & Q1 $(<60)$ & Q2 (60-66) & Q3 (67-73) & Q4 (>73) \\
\hline & Number of participants & 1,044 & 920 & 794 & 908 \\
\hline & Person-years & 24,376 & 21,914 & 19,057 & 20,579 \\
\hline & Number of events & 324 & 270 & 221 & 312 \\
\hline & Crude mortality & 13.3 & 12.3 & 11.6 & 15.2 \\
\hline & Hazard ratio & & & & \\
\hline & Model 1 & 1 & $1.05[0.89,1.23]$ & $0.99[0.83,1.17]$ & $1.24[1.06,1.44]^{* *}$ \\
\hline & Model 2 & 1 & $0.99[0.85,1.17]$ & $0.96[0.81,1.14]$ & $1.18[1.01,1.38]^{*}$ \\
\hline & Model 3 & 1 & $1.02[0.86,1.20]$ & $0.98[0.82,1.16]$ & $1.21[1.03,1.41]^{*}$ \\
\hline \multirow[t]{9}{*}{ Women } & Quantiles (beats/min) & Q1 $(<64)$ & Q2 (64-70) & Q3 (71-77) & $\mathrm{Q} 4(>77)$ \\
\hline & Number of participants & 1,273 & 1,151 & 1,102 & 1,171 \\
\hline & Person-years & 32,002 & 29,402 & 27,637 & 29,159 \\
\hline & Number of events & 256 & 223 & 226 & 241 \\
\hline & Crude mortality & 8.0 & 7.6 & 8.2 & 8.3 \\
\hline & Hazard ratio & & & & \\
\hline & Model 1 & 1 & $1.08[0.91,1.30]$ & $1.12[0.94,1.34]$ & $1.20[1.01,1.43]^{*}$ \\
\hline & Model 2 & 1 & $1.07[0.89,1.28]$ & $1.09[0.91,1.30]$ & $1.14[0.95,1.37]$ \\
\hline & Model 3 & 1 & $1.08[0.90,1.30]$ & $1.12[0.93,1.34]$ & $1.19[1.00,1.43]^{*}$ \\
\hline
\end{tabular}

Model 1 is adjusted for gender (in gender specific model no gender adjusted) and age.

Model 2 is adjusted for gender, age, body mass index (BMI), blood glucose, systolic blood pressure (SBP), hypertension treatment, total-cholesterol, smoking status, and alcohol drinking status.

Model 3 is adjusted for gender, age, BMI, serum albumin (ALB), blood glucose, SBP, hypertension treatment, total-cholesterol, smoking status, and alcohol drinking status.

${ }^{*} P<0.05{ }^{* *} P<0.01{ }^{* * *} P<0.001$ 
Liu Y, et al.

Table 3. Crude mortality rates and hazard ratios for all-cause, cardiovascular disease (CVD) and non-CVD mortality according to resting heart rate (RHR) quantiles in low and high serum albumin (ALB)

\begin{tabular}{|c|c|c|c|c|c|c|c|c|c|}
\hline \multirow{2}{*}{$\begin{array}{l}\text { All-cause mortality } \\
\text { Total } \\
\text { (RHR Quantile in beats/min) }\end{array}$} & \multicolumn{4}{|c|}{ Low albumin } & \multicolumn{5}{|l|}{ High albumin } \\
\hline & $\begin{array}{l}\text { Q1 } \\
(<62)\end{array}$ & $\begin{array}{l}\text { Q2 } \\
(62-68)\end{array}$ & $\begin{array}{l}\text { Q3 } \\
(69-77)\end{array}$ & $\begin{array}{l}\text { Q4 } \\
(>77)\end{array}$ & $\begin{array}{l}\text { Q1 } \\
(<62)\end{array}$ & $\begin{array}{l}\text { Q2 } \\
(62-68)\end{array}$ & $\begin{array}{l}\text { Q3 } \\
(69-77)\end{array}$ & $\begin{array}{l}\text { Q4 } \\
(>77)\end{array}$ & $\begin{array}{l}P \text {-values for } \\
\text { interaction }\end{array}$ \\
\hline Number of participants & 1,007 & 896 & 910 & 797 & 1,218 & 1,153 & 1,228 & 1,154 & \\
\hline Person-years & 21,936 & 31,589 & 20,661 & 30,361 & 20,863 & 31,879 & 17,253 & 29,582 & \\
\hline Number of deaths & 533 & 418 & 427 & 411 & 359 & 315 & 323 & 342 & \\
\hline $\begin{array}{l}\text { Crude mortality rate } / 1,000 \\
\text { person-years } \\
\text { Hazard ratio }\end{array}$ & 24.3 & 13.2 & 20.7 & 13.5 & 17.2 & 9.9 & 18.7 & 11.6 & \\
\hline Model 1 & 1 & $\begin{array}{l}0.82 \\
{[0.72,0.94]^{* *}}\end{array}$ & $\begin{array}{l}0.83 \\
{[0.73,0.94]^{* *}}\end{array}$ & $\begin{array}{l}0.98 \\
{[0.86,1.11]}\end{array}$ & $\begin{array}{l}0.45 \\
{[0.39,0.51]^{* * *}}\end{array}$ & $\begin{array}{l}0.40 \\
{[0.35,0.47]^{* * * *}}\end{array}$ & $\begin{array}{l}0.40 \\
{[0.34,0.45]^{* * * *}}\end{array}$ & $\begin{array}{l}0.45 \\
{[0.40,0.52]^{* * *}}\end{array}$ & \\
\hline Model 2 & 1 & $\begin{array}{l}1.02 \\
{[0.89,1.16]}\end{array}$ & $\begin{array}{l}1.12 \\
{[0.99,1.28]}\end{array}$ & $\begin{array}{l}1.35 \\
{[1.18,1.53]^{* * *}}\end{array}$ & $\begin{array}{l}0.85 \\
{[0.74,0.97]^{*}}\end{array}$ & $\begin{array}{l}0.98 \\
{[0.85,1.13]}\end{array}$ & $\begin{array}{l}0.88 \\
{[0.77,1.02]}\end{array}$ & $\begin{array}{l}0.96 \\
{[0.83,1.10]}\end{array}$ & $P=0.007$ \\
\hline $\begin{array}{l}\text { Men } \\
\text { (RHR Quantile in beats/min) }\end{array}$ & $\begin{array}{l}\text { Q1 } \\
(<60)\end{array}$ & $\begin{array}{l}\text { Q2 } \\
(60-66)\end{array}$ & $\begin{array}{l}\text { Q3 } \\
(67-73)\end{array}$ & $\begin{array}{l}\text { Q4 } \\
(>73)\end{array}$ & $\begin{array}{l}\text { Q1 } \\
(<60)\end{array}$ & $\begin{array}{l}\text { Q2 } \\
(60-66)\end{array}$ & $\begin{array}{l}\text { Q3 } \\
(67-73)\end{array}$ & $\begin{array}{l}\text { Q4 } \\
(>73)\end{array}$ & \\
\hline Number of participants & 424 & 328 & 270 & 325 & 620 & 592 & 524 & 583 & \\
\hline Person-years (total) & 8,350 & 6,483 & 5,486 & 6,048 & 16,025 & 15,430 & 13,570 & 14,530 & \\
\hline Number of deaths & 283 & 220 & 175 & 234 & 190 & 174 & 145 & 210 & \\
\hline $\begin{array}{l}\text { Crude mortality rate } / 1,000 \\
\text { person-years } \\
\text { Hazard ratio }\end{array}$ & 33.9 & 33.9 & 31.9 & 38.7 & 11.9 & 11.3 & 10.7 & 14.5 & \\
\hline Model 1 & 1 & $\begin{array}{l}1.05 \\
{[0.88,1.25]}\end{array}$ & $\begin{array}{l}0.99 \\
{[0.82,1.20]}\end{array}$ & $\begin{array}{l}1.26 \\
{[1.06,1.50]^{* *}}\end{array}$ & $\begin{array}{l}0.78 \\
{[0.64,0.94]^{* *}}\end{array}$ & $\begin{array}{l}0.84 \\
{[0.69,1.02]}\end{array}$ & $\begin{array}{l}0.78 \\
{[0.64,0.96]^{*}}\end{array}$ & $\begin{array}{l}0.94 \\
{[0.78,1.12]}\end{array}$ & \\
\hline Model 2 & 1 & $\begin{array}{l}0.99 \\
{[0.83,1.18]}\end{array}$ & $\begin{array}{l}0.94 \\
{[0.78,1.14]}\end{array}$ & $\begin{array}{l}1.18 \\
{[0.99,1.40]}\end{array}$ & $\begin{array}{l}0.85 \\
{[0.69,1.02]}\end{array}$ & $\begin{array}{l}0.83 \\
{[0.68,1.02]}\end{array}$ & $\begin{array}{l}0.81 \\
{[0.65,0.99]^{*}}\end{array}$ & $\begin{array}{l}0.90 \\
{[0.74,1.08]}\end{array}$ & $P=0.25$ \\
\hline $\begin{array}{l}\text { Women } \\
\text { (RHR Quantile in beats/min) }\end{array}$ & $\begin{array}{l}\text { Q1 } \\
(<64)\end{array}$ & $\begin{array}{l}\text { Q2 } \\
(64-70)\end{array}$ & $\begin{array}{l}\text { Q3 } \\
(71-77) \\
\end{array}$ & $\begin{array}{l}\mathrm{Q} 4 \\
(>77) \\
\end{array}$ & $\begin{array}{l}\text { Q1 } \\
(<64) \\
\end{array}$ & $\begin{array}{l}\text { Q2 } \\
(64-70)\end{array}$ & $\begin{array}{l}\text { Q3 } \\
(71-77) \\
\end{array}$ & $\begin{array}{l}\text { Q4 } \\
(>77) \\
\end{array}$ & \\
\hline Number of participants & 670 & 581 & 504 & 508 & 603 & 570 & 598 & 663 & \\
\hline Person-years (total) & 16,065 & 14,380 & 12,081 & 11,818 & 15,936 & 15,022 & 15,555 & 17,340 & \\
\hline Number of deaths & 257 & 204 & 202 & 214 & 157 & 142 & 155 & 166 & \\
\hline $\begin{array}{l}\text { Crude mortality rate } / 1,000 \\
\text { person-years } \\
\text { Hazard ratio }\end{array}$ & 16.0 & 14.2 & 16.7 & 18.1 & 9.9 & 9.5 & 10.0 & 9.6 & \\
\hline Model 1 & 1 & $\begin{array}{l}1.03 \\
{[0.85,1.24]}\end{array}$ & $\begin{array}{l}1.12 \\
{[0.93,1.35]}\end{array}$ & $\begin{array}{l}1.29 \\
{[1.08,1.55]^{* *}}\end{array}$ & $\begin{array}{l}0.88 \\
{[0.72,1.07]}\end{array}$ & $\begin{array}{l}0.95 \\
{[0.77,1.17]}\end{array}$ & $\begin{array}{l}0.93 \\
{[0.76,1.14]}\end{array}$ & $\begin{array}{l}0.93 \\
{[0.77,1.13]}\end{array}$ & \\
\hline Model 2 & 1 & $\begin{array}{l}1.03 \\
{[0.86,1.23]}\end{array}$ & $\begin{array}{l}1.11 \\
{[0.92,1.34]}\end{array}$ & $\begin{array}{l}1.24 \\
{[1.03,1.49]^{*}}\end{array}$ & $\begin{array}{l}0.89 \\
{[0.73,1.09]}\end{array}$ & $\begin{array}{l}0.95 \\
{[0.77,1.16]}\end{array}$ & $\begin{array}{l}0.90 \\
{[0.74,1.11]}\end{array}$ & $\begin{array}{l}0.87 \\
{[0.71,1.06]}\end{array}$ & $P=0.01$ \\
\hline CVD mortality & Low alb & $\min$ & & & High albumin & & & & \\
\hline $\begin{array}{l}\text { Total } \\
\text { (RHR Quantile in beats/min) }\end{array}$ & $\begin{array}{l}\text { Q1 } \\
(<62)\end{array}$ & $\begin{array}{l}\text { Q2 } \\
(62-68) \\
\end{array}$ & $\begin{array}{l}\text { Q3 } \\
(69-77) \\
\end{array}$ & $\begin{array}{l}\text { Q4 } \\
(>77)\end{array}$ & $\begin{array}{l}\text { Q1 } \\
(<62)\end{array}$ & $\begin{array}{l}\text { Q2 } \\
(62-68) \\
\end{array}$ & $\begin{array}{l}\text { Q3 } \\
(69-77) \\
\end{array}$ & $\begin{array}{l}\text { Q4 } \\
(>77)\end{array}$ & \\
\hline Number of participants & 1,007 & 896 & 910 & 797 & 1,218 & 1,153 & 1,228 & 1,154 & \\
\hline Person-years (total) & 21,936 & 31,589 & 20,661 & 30,361 & 20,863 & 31,879 & 17,253 & 29,582 & \\
\hline Number of deaths & 193 & 139 & 141 & 159 & 122 & 91 & 119 & 91 & \\
\hline $\begin{array}{l}\text { Crude mortality rate } / 1,000 \\
\text { person-years } \\
\text { Hazard ratio }\end{array}$ & 8.8 & 4.4 & 6.8 & 5.2 & 5.8 & 2.9 & 6.9 & 3.1 & \\
\hline Model 1 & 1 & $\begin{array}{l}0.91 \\
{[0.73,1.13]}\end{array}$ & $\begin{array}{l}0.99 \\
{[0.80,1.23]}\end{array}$ & $\begin{array}{l}1.35 \\
{[1.09,1.67]^{* *}}\end{array}$ & $\begin{array}{l}0.92 \\
{[0.73,1.16]}\end{array}$ & $\begin{array}{l}0.89 \\
{[0.69,1.16]}\end{array}$ & $\begin{array}{l}0.98 \\
{[0.77,1.23]}\end{array}$ & $\begin{array}{l}0.75 \\
{[0.58,0.96]^{*}}\end{array}$ & \\
\hline Model 2 & 1 & $\begin{array}{l}0.87 \\
{[0.70,1.09]}\end{array}$ & $\begin{array}{l}0.95 \\
{[0.76,1.19]}\end{array}$ & $\begin{array}{l}1.27 \\
{[1.02,1.57]^{*}}\end{array}$ & $\begin{array}{l}0.95 \\
{[0.76,1.20]}\end{array}$ & $\begin{array}{l}0.83 \\
{[0.64,1.07]}\end{array}$ & $\begin{array}{l}0.88 \\
{[0.70,1.12]}\end{array}$ & $\begin{array}{l}0.61 \\
{[0.47,0.79]^{* * * *}}\end{array}$ & $P<0.001$ \\
\hline $\begin{array}{l}\text { Men } \\
\text { (RHR Quantile in beats/min) }\end{array}$ & $\begin{array}{l}\text { Q1 } \\
(<60)\end{array}$ & $\begin{array}{l}\text { Q2 } \\
(60-66)\end{array}$ & $\begin{array}{l}\text { Q3 } \\
(67-73)\end{array}$ & $\begin{array}{l}\text { Q4 } \\
(>73)\end{array}$ & $\begin{array}{l}\text { Q1 } \\
(<60)\end{array}$ & $\begin{array}{l}\text { Q2 } \\
(60-66)\end{array}$ & $\begin{array}{l}\text { Q3 } \\
(67-73)\end{array}$ & $\begin{array}{l}\text { Q4 } \\
(>73)\end{array}$ & \\
\hline Number of participants & 424 & 328 & 270 & 325 & 620 & 592 & 524 & 583 & \\
\hline Person-years (total) & 8,350 & 6,483 & 5,486 & 6,048 & 16,025 & 15,430 & 13,570 & 14,530 & \\
\hline Number of deaths & 283 & 220 & 175 & 234 & 190 & 174 & 145 & 210 & \\
\hline $\begin{array}{l}\text { Crude mortality rate } / 1,000 \\
\text { person-years } \\
\text { Hazard ratio }\end{array}$ & 33.9 & 33.9 & 31.9 & 38.7 & 11.9 & 11.3 & 10.7 & 14.5 & \\
\hline Model 1 & 1 & $\begin{array}{l}1.14 \\
{[0.84,1.55]}\end{array}$ & $\begin{array}{l}0.92 \\
{[0.65,1.30]}\end{array}$ & $\begin{array}{l}1.32 \\
{[0.97,1.79]}\end{array}$ & $\begin{array}{l}0.93 \\
{[0.67,1.30]}\end{array}$ & $\begin{array}{l}0.92 \\
{[0.64,1.31]}\end{array}$ & $\begin{array}{l}1.01 \\
{[0.71,1.45]}\end{array}$ & $\begin{array}{l}0.91 \\
{[0.65,1.28]}\end{array}$ & \\
\hline Model 2 & 1 & $\begin{array}{l}1.07 \\
{[0.78,1.45]}\end{array}$ & $\begin{array}{l}0.85 \\
{[0.60,1.20]}\end{array}$ & $\begin{array}{l}1.18 \\
{[0.87,1.61]}\end{array}$ & $\begin{array}{l}1.00 \\
{[0.71,1.41]}\end{array}$ & $\begin{array}{l}0.84 \\
{[0.59,1.21]}\end{array}$ & $\begin{array}{l}0.97 \\
{[0.67,1.40]}\end{array}$ & $\begin{array}{l}0.75 \\
{[0.53,1.07]}\end{array}$ & $P=0.25$ \\
\hline $\begin{array}{l}\text { Women } \\
\text { (RHR Quantile in beats/min) }\end{array}$ & $\begin{array}{l}\text { Q1 } \\
(<64)\end{array}$ & $\begin{array}{l}\text { Q2 } \\
(64-70)\end{array}$ & $\begin{array}{l}\text { Q3 } \\
(71-77)\end{array}$ & $\begin{array}{l}\text { Q4 } \\
(>77)\end{array}$ & $\begin{array}{l}\text { Q1 } \\
(<64)\end{array}$ & $\begin{array}{l}\text { Q2 } \\
(64-70)\end{array}$ & $\begin{array}{l}\text { Q3 } \\
(71-77)\end{array}$ & $\begin{array}{l}\text { Q4 } \\
(>77)\end{array}$ & \\
\hline Number of participants & 670 & 581 & 504 & 508 & 603 & 570 & 598 & 663 & \\
\hline Person-years (total) & 16,065 & 14,380 & 12,081 & 11,818 & 15,936 & 15,022 & 15,555 & 17,340 & \\
\hline Number of deaths & 257 & 204 & 202 & 214 & 157 & 142 & 155 & 166 & \\
\hline
\end{tabular}




\begin{tabular}{|c|c|c|c|c|c|c|c|c|c|}
\hline $\begin{array}{l}\text { Women } \\
\text { (RHR Quantile in beats/min) }\end{array}$ & $\begin{array}{l}\text { Q1 } \\
(<64)\end{array}$ & $\begin{array}{l}\text { Q2 } \\
(64-70)\end{array}$ & $\begin{array}{l}\text { Q3 } \\
(71-77)\end{array}$ & $\begin{array}{l}\text { Q4 } \\
(>77)\end{array}$ & $\begin{array}{l}\text { Q1 } \\
(<64)\end{array}$ & $\begin{array}{l}\text { Q2 } \\
(64-70)\end{array}$ & $\begin{array}{l}\text { Q3 } \\
(71-77)\end{array}$ & $\begin{array}{l}\text { Q4 } \\
(>77)\end{array}$ & \\
\hline $\begin{array}{l}\text { Crude mortality rate } / 1,000 \\
\text { person-years }\end{array}$ & 16.0 & 14.2 & 16.7 & 18.1 & 9.9 & 9.5 & 10.0 & 9.6 & \\
\hline Hazard ratio & & & & & & & & & \\
\hline Model 1 & 1 & $\begin{array}{l}0.97 \\
{[0.72,1.31]}\end{array}$ & $\begin{array}{l}0.96 \\
{[0.71,1.30]}\end{array}$ & $\begin{array}{l}1.32 \\
{[0.99,1.75]}\end{array}$ & $\begin{array}{l}0.85 \\
{[0.62,1.19]}\end{array}$ & $\begin{array}{l}0.86 \\
{[0.61,1.22]}\end{array}$ & $\begin{array}{l}0.95 \\
{[0.69,1.31]}\end{array}$ & $\begin{array}{l}0.72 \\
{[0.51,1.02]}\end{array}$ & \\
\hline Model 2 & 1 & $\begin{array}{l}0.97 \\
{[0.72,1.32]}\end{array}$ & $\begin{array}{l}0.98 \\
{[0.72,1.33]}\end{array}$ & $\begin{array}{l}1.32 \\
{[0.98,1.76]}\end{array}$ & $\begin{array}{l}0.83 \\
{[0.60,1.16]}\end{array}$ & $\begin{array}{l}0.82 \\
{[0.58,1.17]}\end{array}$ & $\begin{array}{l}0.88 \\
{[0.64,1.22]}\end{array}$ & $\begin{array}{l}0.59 \\
{[0.42,0.85]^{* *}}\end{array}$ & $P=0.002$ \\
\hline Non-CVD death & \multicolumn{4}{|c|}{ Low albumin } & \multicolumn{4}{|l|}{ High albumin } & \\
\hline $\begin{array}{l}\text { Total } \\
\text { (RHR Quantile in beats/min) }\end{array}$ & $\begin{array}{l}\text { Q1 } \\
(<62)\end{array}$ & $\begin{array}{l}\text { Q2 } \\
(62-68)\end{array}$ & $\begin{array}{l}\text { Q3 } \\
(69-77)\end{array}$ & $\begin{array}{l}\text { Q4 } \\
(>77)\end{array}$ & $\begin{array}{l}\text { Q1 } \\
(<62)\end{array}$ & $\begin{array}{l}\text { Q2 } \\
(62-68)\end{array}$ & $\begin{array}{l}\text { Q3 } \\
(69-77)\end{array}$ & $\begin{array}{l}\text { Q4 } \\
(>77)\end{array}$ & \\
\hline Number of participants & 1,007 & 896 & 910 & 797 & 1,218 & 1,153 & 1,228 & 1,154 & \\
\hline Person-years (total) & 21,936 & 31,589 & 20,661 & 30,361 & 20,863 & 31,879 & 17,253 & 29,582 & \\
\hline Number of deaths & 340 & 279 & 286 & 252 & 237 & 224 & 204 & 251 & \\
\hline $\begin{array}{l}\text { Crude mortality rate } / 1,000 \\
\text { person-years } \\
\text { Hazard ratio }\end{array}$ & 15.5 & 8.8 & 13.8 & 8.3 & 11.4 & 7.0 & 11.8 & 8.5 & \\
\hline Model 1 & 1 & $\begin{array}{l}1.08 \\
{[0.92,1.27]}\end{array}$ & $\begin{array}{l}1.20 \\
{[1.02,1.40]^{*}}\end{array}$ & $\begin{array}{l}1.33 \\
{[1.13,1.56]^{* * *}}\end{array}$ & $\begin{array}{l}0.82 \\
{[0.69,0.97]^{*}}\end{array}$ & $\begin{array}{l}1.02 \\
{[0.86,1.21]}\end{array}$ & $\begin{array}{l}0.83 \\
{[0.70,0.99]^{*}}\end{array}$ & $\begin{array}{l}1.06 \\
{[0.90,1.25]}\end{array}$ & \\
\hline Model 2 & 1 & {$[0.91,1.25]$} & $\begin{array}{l}1.16 \\
{[0.99,1.36]}\end{array}$ & $\begin{array}{l}1.27 \\
{[1.07,1.50]^{* *}}\end{array}$ & $\begin{array}{l}0.87 \\
{[0.73,1.03]}\end{array}$ & $\begin{array}{l}1.05 \\
{[0.88,1.25]}\end{array}$ & $\begin{array}{l}0.85 \\
{[0.71,1.02]}\end{array}$ & $\begin{array}{l}1.05 \\
{[0.89,1.24]}\end{array}$ & $P=0.31$ \\
\hline $\begin{array}{l}\text { Men } \\
\text { (RHR Quantile in beats/min) }\end{array}$ & $\begin{array}{l}\text { Q1 } \\
(<60)\end{array}$ & $\begin{array}{l}\text { Q2 } \\
(60-66)\end{array}$ & $\begin{array}{l}\text { Q3 } \\
(67-73) \\
\end{array}$ & $\begin{array}{l}\text { Q4 } \\
(>73)\end{array}$ & $\begin{array}{l}\text { Q1 } \\
(<60)\end{array}$ & $\begin{array}{l}\text { Q2 } \\
(60-66)\end{array}$ & $\begin{array}{l}\text { Q3 } \\
(67-73) \\
\end{array}$ & $\begin{array}{l}\text { Q4 } \\
(>73)\end{array}$ & \\
\hline Number of participants & 424 & 328 & 270 & 325 & 620 & 592 & 524 & 583 & \\
\hline Person-years (total) & 8,350 & 6,483 & 5,486 & 6,048 & 16,025 & 15,430 & 13,570 & 14,530 & \\
\hline Number of deaths & 89 & 75 & 51 & 77 & 60 & 49 & 48 & 55 & \\
\hline $\begin{array}{l}\text { Crude mortality rate } / 1,000 \\
\text { person-years } \\
\text { Hazard ratio }\end{array}$ & 10.7 & 11.6 & 9.3 & 12.7 & 3.7 & 3.2 & 3.5 & 3.8 & \\
\hline Model 1 & 1 & $\begin{array}{l}1.01 \\
{[0.81,1.25]}\end{array}$ & $\begin{array}{l}1.02 \\
{[0.82,1.28]}\end{array}$ & $\begin{array}{l}1.24 \\
{[1.01,1.53]^{*}}\end{array}$ & $\begin{array}{l}0.72 \\
{[0.57,0.90]^{* *}}\end{array}$ & $\begin{array}{l}0.80 \\
{[0.63,1.01]}\end{array}$ & $\begin{array}{l}0.70 \\
{[0.54,0.90]^{* *}}\end{array}$ & $\begin{array}{l}0.94 \\
{[0.75,1.16]}\end{array}$ & \\
\hline Model 2 & 1 & $\begin{array}{l}0.96 \\
{[0.77,1.20]}\end{array}$ & $\begin{array}{l}0.99 \\
{[0.78,1.24]} \\
\end{array}$ & $\begin{array}{l}1.18 \\
{[0.95,1.46]}\end{array}$ & $\begin{array}{l}0.79 \\
{[0.62,0.99]^{*}}\end{array}$ & $\begin{array}{l}0.83 \\
{[0.66,1.05]}\end{array}$ & $\begin{array}{l}0.75 \\
{[0.58,0.97]^{*}}\end{array}$ & $\begin{array}{l}0.97 \\
{[0.77,1.21]}\end{array}$ & $P=0.52$ \\
\hline $\begin{array}{l}\text { Women } \\
\text { (RHR Quantile in beats/min) }\end{array}$ & $\begin{array}{l}\text { Q1 } \\
(<64)\end{array}$ & $\begin{array}{l}\text { Q2 } \\
(64-70)\end{array}$ & $\begin{array}{l}\text { Q3 } \\
(71-77)\end{array}$ & $\begin{array}{l}\text { Q4 } \\
(>77)\end{array}$ & $\begin{array}{l}\text { Q1 } \\
(<64)\end{array}$ & $\begin{array}{l}\text { Q2 } \\
(64-70)\end{array}$ & $\begin{array}{l}\text { Q3 } \\
(71-77)\end{array}$ & $\begin{array}{l}\text { Q4 } \\
(>77)\end{array}$ & \\
\hline Number of participants & 670 & 581 & 504 & 508 & 603 & 570 & 598 & 663 & \\
\hline Person-years (total) & 16,065 & 14,380 & 12,081 & 11,818 & 15,936 & 15,022 & 15,555 & 17,340 & \\
\hline Number of deaths & 155 & 128 & 131 & 123 & 101 & 95 & 95 & 118 & \\
\hline $\begin{array}{l}\text { Crude mortality rate } / 1,000 \\
\text { person-years } \\
\text { Hazard ratio }\end{array}$ & 9.6 & 8.9 & 10.8 & 10.4 & 6.3 & 6.3 & 6.1 & 6.8 & \\
\hline Model 1 & 1 & $\begin{array}{l}1.07 \\
{[0.84,1.35]}\end{array}$ & $\begin{array}{l}1.22 \\
{[0.96,1.54]}\end{array}$ & $\begin{array}{l}1.25 \\
{[0.99,1.59]}\end{array}$ & $\begin{array}{l}0.89 \\
{[0.70,1.15]}\end{array}$ & $\begin{array}{l}1.01 \\
{[0.78,1.30]}\end{array}$ & $\begin{array}{l}0.92 \\
{[0.71,1.19]}\end{array}$ & $\begin{array}{l}1.06 \\
{[0.83,1.35]}\end{array}$ & \\
\hline Model 2 & 1 & {$[0.83,1.33]$} & $\begin{array}{l}1.19 \\
{[0.94,1.51]}\end{array}$ & $\begin{array}{l}1.18 \\
{[0.93,1.50]}\end{array}$ & $\begin{array}{l}0.93 \\
{[0.73,1.20]}\end{array}$ & $\begin{array}{l}1.02 \\
{[0.79,1.33]}\end{array}$ & $\begin{array}{l}0.92 \\
{[0.71,1.19]}\end{array}$ & $\begin{array}{l}1.05 \\
{[0.82,1.34]}\end{array}$ & $P=0.46$ \\
\hline
\end{tabular}

Model 1 is adjusted for gender (in gender specific model no gender adjusted) and age.

Model 2 is adjusted for gender, age, body mass index (BMI), blood glucose, systolic blood pressure (SBP), hypertension treatment, total-cholesterol, smoking status, and alcohol drinking status.

$P$-values for the interaction were those between resting heart rate (continuous) and serum albumin groups.

${ }^{*} P<0.05^{* *} P<0.01{ }^{* * *} P<0.001$

increased risk for CVD mortality among individuals with low ALB levels. Meanwhile, in those with high ALB levels, a higher RHR was correlated with a lower risk for CVD mortality. A positive association was observed between RHR and non-CVD mortality, and a higher RHR was significantly associated with a greater risk among individuals with low ALB levels. However, this result was not observed among individuals with high ALB levels. Thus, the effect of RHR on outcomes, particular on CVD mortality, differed according to ALB levels.

In the current study, we did not observe a direct association between a high RHR and CVD mortality in all participants. In previous cohort studies, meanwhile, RHR was found to be associated with an increased risk for CVD and all-cause mortality in the general population. ${ }^{10,11,24}$ As we previously reported, an elevated RHR was found to be correlated with cardiovascular or all-cause mortality in a 16.5-year follow-up. However, it was only observed in participants aged $30-59$ years. ${ }^{23}$ In previous cohort studies on a Japanese general population with a mean age of about 45 years at baseline, a higher RHR was associated with the development of obesity and diabetes within 20 years $^{25}$ and hypertension within 3 years. ${ }^{26}$ These findings suggest that the activation of the sympathetic nervous system correlated with a high RHR might play a role in the development of these conditions in relatively younger populations. Furthermore, participants with an activated sympathetic nervous system may develop adverse events in the early follow-up period.

Regarding the effect of reducing RHR, previous studies have reported that all-cause mortality can be reduced in patients with 
HF by lowering RHR with the use of medications, such as betablockers. ${ }^{27}$ However, no findings showed the additional value of RHR-lowering therapy with beta-blockers in hypertensive patients without CVD. ${ }^{10,11,24}$ In a consensus conference wherein recommendations for the management of hypertensive patients with increased RHR were updated, gathering panelists reconfirmed that RHR-lowering therapy had no additional benefit for tachycardia hypertension. They recommended that further research should be performed to validate unresolved discrepancies between the findings of cohort studies and clinical trials. ${ }^{28}$ Accordingly, the effect of RHR on mortality is controversial, which might be caused by the characteristics of participants.

In our analysis stratified according to ALB levels, a higher RHR was significantly associated with CVD mortality and nonCVD mortality among individuals with low ALB levels. Since ALB is a marker of anti-inflammatory, antioxidant, anticoagulant, and antiplatelet aggregation activities, as well as nutritional status, it might be correlated with the risk for mortality. ${ }^{29}$ In the present study, individuals with lower ALB levels could present with subclinical changes in health status, although they might not experience actual malnutrition. However, the protein intake proportion showed no significant difference between ALB groups (mean 15.30; standard deviation [SD], $2.12 \mathrm{~g} / 1,000 \mathrm{kcal}$ in low ALB vs mean 15.27; SD, $2.10 \mathrm{~g} / 1,000 \mathrm{kcal}$ in high ALB) in the present study, a significant difference was shown in total energy intake (mean 2,063; SD, $491 \mathrm{kcal} /$ day in low ALB vs mean 2,193; SD, $510 \mathrm{kcal} /$ day in high ALB, $P<0.001)$. Hypoalbuminemia is associated with an increased risk of death in HF patients with reduced ejection fraction, in whom the magnitude of heart rate reduction is associated with the survival benefit of betablockers. ${ }^{30}$ Moreover, ALB is associated with myocardial fibrosis, adverse pulsatile aortic hemodynamics, and prognosis in $\mathrm{HF}$ patients with preserved ejection fraction. ${ }^{31}$ There are individuals with latent $\mathrm{HF}$ even in the general population. ${ }^{32} \mathrm{ALB}$ has protective effects against low-density lipoprotein cholesterol from $\mathrm{Cu} 2+$ oxidation, which is a crucial step for atherogenesis, in addition to $\mathrm{HF}^{33}$ Glycated or oxidated ALB can no longer hold copper ion. ${ }^{34}$ Abnormal ALB levels can decrease the ALB/ high-sensitivity $\mathrm{C}$-reactive protein ratio, and this proinflammation imbalance can result in the development or worsening of coronary slow flow. ${ }^{35}$ Overall, a normal ALB level is crucial for preventing CVD, and low ALB levels caused by high oxidative levels and glycation stress disrupt anti-atherogenesis functioning. Such a strong effect might account for the impact of RHR. Accordingly, individuals with health impairment might be at risk for a high RHR, which is considered a marker of mortality. Also, if we take a deeper subgroup analysis for cause-specific mortality in CVD, the cerebral infarction made a great contribution to it (eTable 2). In this analysis, ALB level differently impacted males and females according to RHR quantile, which could explain why RHR quantile elevation caused both increase and decrease in ALB in the stratified CVD mortality model. This is similar to a previous study in Japan. ${ }^{36}$

On the contrary, in the present study, a lower risk in higher RHR was shown among individuals with high ALB. This result might be controversial compared to previous evidence. Actually, there is a little physiological evidence supporting our results. Some studies have shown that lower RHR was associated with left ventricular hypertrophy $(\mathrm{LVH})$ progression. ${ }^{37,38} \mathrm{LVH}$ is a risk factor for CVD events and all-cause mortality even in a general population. The present study might suggest that lower RHR among individuals with high ALB increases risk of CVD mortality mediated by potential LVH progression. We conducted an additional analysis considering recorded findings of left high R-wave (1-high-R) on electrocardiogram, which was performed as the National Survey on Circulatory Disorders in 1980. Participants with lower RHR had higher proportion of those with 1-high-R. However, the association between RHR and CVD mortality was almost unchanged after adjusting for 1-high-R, irrespective of ALB levels. Another explanation is that, while elevated heart rate was associated with increased peripheral blood pressure, there was an inverse relationship between resting heart rate and augmentation index, ${ }^{39}$ and elevated augmentation index and consequently increased central blood pressure were risk factors for CVD events. ${ }^{40,41}$ Particularly, the inverse association was more apparent in higher level of aortic stiffness among normotensive and untreated hypertensive participants. ${ }^{42}$ Furthermore, in the CAFÉ study, ${ }^{37}$ the use of a beta-blocker was associated with RHR reduction and central aortic pressure elevation simultaneously, despite similar brachial systolic blood pressure. These findings suggest that RHR might reflect central hemodynamics, which relate to CVD events. Additionally, when we take a closer look to cause-specific CVD mortality influenced by RHR at high ALB, they were mostly from CHD and cerebral infarction, sequelae of atherosclerotic cardiovascular disease. As described earlier, ALB has anti-atherosclerotic properties, such as anti-inflammatory and antioxidant effects, as well as being a marker of nutritional status; therefore, individuals with relatively higher ALB would be protected against atherosclerosis. Accordingly, we speculate that RHR at high ALB has a different impact on CVD from that of low ALB. However, there is little evidence on the relationship between RHR and ALB, which may include many unexamined confounding factors, such as socioeconomic status and mental stress. Although the background on the results is not clear, we believe our results are important because this is a first finding, at least to our knowledge. Future studies are needed to clarify the clinical significance of RHR.

The current study had several limitations. First, we can only adjust some basic confounders in mortality models. Other potential risk factors, such as thyroid hormone function, physical activity, high-density lipoprotein cholesterol, and inflammatory marker levels could not be adjusted in the statistical models. Second, since this study was based on risk factor measurement on one occasion only, our findings did not reflect changes in RHR and confounding risk factors, such as blood pressure and serum glucose level during follow-up. Third, we did not have a precise information on the treatment of hypertension; thus, participants who used beta-blockers were not identified. Finally, we measured RHR via electrocardiogram during daytime, and data on RHR obtained during nighttime or daytime immediately after waking were not available. A follow-up study on six populations in several countries, including Japan, used the RHR obtained during office measurements, and data on 24-h ambulatory blood pressure were used. Results showed that morning and nighttime, not daytime, RHRs were associated with CVD mortality. ${ }^{37}$

\section{Conclusion}

The impact of RHR on CVD mortality differed according to ALB levels in a general Japanese population. Such an effect might depend on health status, including nutrition. Thus, further studies must be conducted to elucidate the underlying mechanism. 


\section{ACKNOWLEDGEMENTS}

The authors would like to thank staff members of the NIPPON DATA80/90 Research Group. The NIPPON DATA80/90 Research Group is composed of the following investigators. Chairpersons: Hirotsugu Ueshima (Center for Epidemiologic Research in Asia, Shiga University of Medical Science, Otsu, Shiga), Akira Okayama (Research Institute of Strategy for Prevention, Tokyo) for the NIPPON DATA80; Hirotsugu Ueshima, Tomonori Okamura (Department of Preventive Medicine and Public Health, Keio University, Tokyo) for the NIPPON DATA90. Research members: Shigeyuki Saitoh (School of Health Sciences, Sapporo Medical University, Sapporo, Hokkaido), Kiyomi Sakata (Department of Hygiene and Preventive Medicine, Iwate Medical University, Morioka, Iwate), Atsushi Hozawa (Department of Preventive Medicine and Epidemiology, Tohoku Medical Megabank Organization, Tohoku University, Sendai, Miyagi), Yosikazu Nakamura (Department of Public Health, Jichi Medical University, Shimotsuke, Tochigi), Nobuo Nishi (Center for International Collaboration and Partnership, National Institutes of Biomedical Innovation, Health and Nutrition, Tokyo), Nagako Okuda (Department of Health and Nutrition, University of Human Arts and Sciences, Saitama), Takayoshi Ohkubo (Department of Hygiene and Public Health Teikyo University School of Medicine, Tokyo), Fumiyoshi Kasagi (Institute of Radiation Epidemiology, Radiation Effects Association, Tokyo), Yoshitaka Murakami (Department of Medical Statistics, Toho University, Tokyo), Toru Izumi (Kitasato University, Sagamihara, Kanagawa), Yasuhiro Matsumura (Faculty of Health and Nutrition, Bunkyo University, Chigasaki, Kanagawa), Toshiyuki Ojima (Department of Community Health and Preventive Medicine, Hamamatsu University School of Medicine, Hamamatsu, Shizuoka), Koji Tamakoshi (Department of Public Health and Health Information Dynamics, Nagoya University Graduate School of Medicine, Nagoya, Aichi), Hideaki Nakagawa (Medical Research Institute, Kanazawa Medical University, Kanazawa, Ishikawa), Yoshikuni Kita (Faculty of Nursing Science, Tsuruga Nursing University, Tsuruga, Fukui), Katsuyuki Miura (Director of the Center for Epidemiologic Research in Asia, Department of Public Health, Shiga University of Medical Science, Otsu, Shiga), Aya Kadota (Center for Epidemiologic Research in Asia, Shiga University of Medical Science, Otsu, Shiga), Akira Fujiyoshi (Department of Public Health, Shiga University of Medical Science, Otsu, Shiga), Naomi Miyamatsu (Department of Clinical Nursing, Shiga University of Medical Science, Otsu, Shiga), Yasuyuki Nakamura (Department of Food Science and Human Nutrition, Ryukoku University, Otsu, Shiga), Takehito Hayakawa (Kinugasa Research Organization, Ritsumeikan University, Kyoto), Katsushi Yoshita (Osaka City University Graduate School of human life science, Osaka), Yoshihiro Miyamoto (Department of Preventive Cardiology, National Cerebral and Cardiovascular Center, Suita, Osaka), Kazunori Kodama (Radiation Effects Research Foundation, Hiroshima) and Yutaka Kiyohara (Hisayama Research Institute for Lifestyle Discascs, Hisayamacho, Fukuoka).

Funding: This study was supported by a Grant-in-Aid from the Ministry of Health, Labour and Welfare under the auspices of the Japanese Association for Cerebro-cardiovascular Disease Control, Research Grant for Cardiovascular Diseases (7A-2) from the Ministry of Health, Labour and Welfare, and Health and Labour
Sciences Research Grants, Japan (Comprehensive Research on Aging and Health [H11-Chouju-046, H14-Chouju-003, H17Chouju-012, H19-Chouju-Ippan-014] and Comprehensive Research on Life-Style Related Diseases including Cardiovascular Diseases and Diabetes Mellitus [H22-Junkankitou-SeishuuSitei-017, H25-Junkankitou-Seishuu-Sitei-022, H30-JunkankitouSitei-002, 21FA2002]).

Conflicts of interest: None declared.

\section{APPENDIX A. SUPPLEMENTARY DATA}

Supplementary data related to this article can be found at https:// doi.org/10.2188/jea.JE20210114.

\section{REFERENCES}

1. Cooney MT, Vartiainen E, Laatikainen T, Juolevi A, Dudina A, Graham IM. Elevated resting heart rate is an independent risk factor for cardiovascular disease in healthy men and women. Am Heart J. $2010 \mathrm{Jul} ; 160(1): 208$.

2. Huang YQ, Shen G, Huang JY, Zhang B, Feng YQ. A nonlinear association between resting heart rate and ischemic stroke among community elderly hypertensive patients. Postgrad Med. 2020; 132(2):215-219.

3. Parikh KS, Greiner MA, Suzuki T, et al. Resting heart rate and longterm outcomes among the African American population insights from the Jackson Heart Study. JAMA Cardiol. 2017;2(2):172-180.

4. Nwabuo CC, Appiah D, Moreira HT, et al. Temporal changes in resting heart rate, left ventricular dysfunction, heart failure and cardiovascular disease: CARDIA Study. Am J Med. 2020;133(8): 946-953.

5. Zhang $\mathrm{M}$, Han $\mathrm{C}$, Wang $\mathrm{C}$, et al. Association of resting heart rate and cardiovascular disease mortality in hypertensive and normotensive rural Chinese. J Cardiol. 2017;69(5):779-784.

6. Chen XJ, Barywani SB, Hansson PO, et al. Impact of changes in heart rate with age on all-cause death and cardiovascular events in 50-year-old men from the general population. Open Heart. 2019; 6(1):e000856.

7. Custodis F, Roggenbuck U, Lehmann N, et al. Resting heart rate is an independent predictor of all-cause mortality in the middle aged general population. Clin Res Cardiol. 2016;105(7):601-612.

8. Münzel T, Hahad O, Gori T, et al. Heart rate, mortality, and the relation with clinical and subclinical cardiovascular diseases: results from the Gutenberg Health Study. Clin Res Cardiol. 2019;108(12): 1313-1323.

9. Palatini P, Benetos A, Grassi G, et al. Identification and management of the hypertensive patient with elevated heart rate: statement of a European Society of Hypertension. J Hypertens. 2006;24(4):603610.

10. Palatini P. Role of elevated heart rate in the development of cardiovascular disease in hypertension. Hypertension. 2011;58(5): $745-750$.

11. Bangalore S, Messerli FH, Kostis JB, Pepine CJ. Cardiovascular protection using beta-blockers: a critical review of the evidence. J Am Coll Cardiol. 2007;50(7):563-572.

12. Hara K, Floras JS. After-effects of exercise on haemodynamics and muscle sympathetic nerve activity in young patients with dilated cardiomyopathy. Heart. 1996;75(6):602-608.

13. Carter JR, Kupiers NT, Ray CA. Neurovascular responses to mental stress. J Physiol. 2005;564(Pt 1):321-327.

14. Catai AM, Chacon-Mikahil MP, Martinelli FS, et al. Effects of aerobic exercise training on heart rate variability during wakefulness and sleep and cardiorespiratory responses of young and middle-aged healthy men. Braz. J Med Biol Res. 2002;35(6):741-752.

15. Ota A, Kondo N, Murayama N, Tanabe N, Shobugawa Y, Kondo K; Japan Gerontological Evaluation Study (JAGES) group. Serum albumin levels and economic status in Japanese older adults. PLoS 
One. 2016;11(6): $\mathrm{e} 0155022$.

16. Hogg N. Biological chemistry and clinical potential of S-nitrosothiols. Free Radic Biol Med. 2000;28(10):1478-1486.

17. Johnson TA, Stasko NA, Matthews JL, et al. Reduced ischemia/ reperfusion injury via glutathione-initiated nitric oxide-releasing dendrimers. Nitric Oxide. 2010;22(1):30-36.

18. Arques S. Human serum albumin in cardiovascular diseases. Eur $J$ Intern Med. 2018;52:8-12.

19. Sajadieh A, Nielsen OW, Rasmussen V, Hein HO, Abedini S, Hansen JF. Increased heart rate and reduced heart-rate variability are associated with subclinical inflammation in middle-aged and elderly subjects with no apparent heart disease. Eur Heart J. 2004;25(5): 363-370.

20. Park SK, Tucker KL, O’Neill MS, et al. Fruit, vegetable, and fish consumption and heart rate variability: the Veterans Administration Normative Aging Study. Am J Clin Nutr. 2009;89(3):778-786.

21. Hisamatsu T, Miura K, Ohkubo T, et al. High long-chain n-3 fatty acid intake attenuates the effect of high resting heart rate on cardiovascular mortality risk: a 24-year follow-up of Japanese general population. J Cardiol. 2014;64(3):218-224.

22. Okamura T, Hayakawa T, Kadowaki T, et al; NIPPON DATA80 research group. A combination of serum low albumin and aboveaverage cholesterol level was associated with excess mortality. J Clin Epidemiol. 2004;57(11):1188-1195.

23. Okamura T, Hayakawa T, Kadowaki T, et al; NIPPONDATA80 Research Group. Resting heart rate and cause-specific death in a 16.5-year cohort study of the Japanese general population. Am Heart J. 2004;147(6):1024-1032.

24. Law MR, Morris JK, Wald NJ. Use of blood pressure lowering drugs in the prevention of cardiovascular disease: meta-analysis of 147 randomised trials in the context of expectations from prospective epidemiological studies. BMJ. 2009;338:b1665.

25. Shigetoh Y, Adachi H, Yamagishi S, et al. Higher heart rate may predispose to obesity and diabetes mellitus: 20-year prospective study in a general population. Am J Hypertens. 2009;22(2):151-155.

26. Inoue T, Iseki K, Iseki C, Kinjo K, Ohya Y, Takishita S. Higher heart rate predicts the risk of developing hypertension in a normotensive screened cohort. Circ J. 2007;71(11):1755-1760.

27. McAlister FA, Wiebe N, Ezekowitz JA, Leung AA, Armstrong PW. Meta-analysis: beta-blocker dose, heart rate reduction, and death in patients with heart failure. Ann Intern Med. 2009;150(11):784-794.

28. Palatini P, Rosei EA, Casiglia E, et al. Management of the hypertensive patient with elevated heart rate: Statement of the Second Consensus Conference endorsed by the European Society of Hypertension. J Hypertens. 2016;34(5):813-821.

29. Brown-Tortorici AR, Naderi N, Tang Y, et al. Serum albumin is incrementally associated with increased mortality across varying levels of kidney function. Nutrition. 2020;79-80:110818.

30. Horwich TB, Kalantar-Zadeh K, MacLellan RW, Fonarow GC.
Albumin levels predict survival in patients with systolic heart failure. Am Heart J. 2008;155(5):883-889.

31. Prenner SB, Pillutla R, Yenigalla S, et al. Serum albumin is a marker of myocardial fibrosis, adverse pulsatile aortic hemodynamics, and prognosis in heart failure with preserved ejection fraction. $J$ Am Heart Assoc. 2020;9(3):e014716.

32. Kanda H, Kita Y, Okamura T, et al. What factors are associated with high plasma B-type natriuretic peptide levels in a general Japanese population? J Hum Hypertens. 2005;19(2):165-172.

33. Kontush A, Chantepie S, Chapman MJ. Small, dense HDL particles exert potent protection of atherogenic LDL against oxidative stress. Arterioscler Thromb Vasc Biol. 2003;23(10):1881-1888.

34. Pinchuk I, Lichtenberg D. The mechanism of action of antioxidants against lipoprotein peroxidation, evaluation based on kinetic experiments. Prog Lipid Res. 2002;41(4):279-314.

35. Kayapinar O, Ozde C, Kaya A. Relationship between the reciprocal change in inflammation-related biomarkers (fibrinogen-to-albumin and hsCRP-to-albumin ratios) and the presence and severity of coronary slow flow. Clin Appl Thromb Hemost. 2019;25: 1076029619835383.

36. Umeki Y, Adachi H, Enomoto M, et al. Serum albumin and Cerebrocardiovascular mortality during a 15 -year study in a communitybased cohort in Tanushimaru, a cohort of the seven countries study. Intern Med. 2016;55(20):2917-2925.

37. Williams B, Lacy PS, Thom SM, et al; CAFE Investigators; AngloScandinavian Cardiac Outcomes Trial Investigators; CAFE Steering Committee and Writing Committee. Differential impact of blood pressure-lowering drugs on central aortic pressure and clinical outcomes: principal results of the Conduit Artery Function Evaluation (CAFE) study. Circulation. 2006;113(9):1213-1225.

38. Inoue $\mathrm{T}$, Arima $\mathrm{H}$, Katsuimata $\mathrm{Y}$, et al. Development of electrocardiographic left ventricular hypertrophy and resting heart rate over time: findings from the OGHMA Study. Angiology. 2020; 71(1):70-76.

39. Reule S, Drawz PE. Heart rate and blood pressure: any possible implications for management of hypertension? Curr Hypertens Rep. 2012;14(6):478-484.

40. London GM, Blacher J, Pannier B, et al. Arterial wave reflections and survival in end-stage renal failure. Hypertension. 2001;38(3): 434-438.

41. Protogerou AD, Papaioannou TG, Blacher J, et al. Central blood pressures: do we need them in the management of cardiovascular disease? Is it a feasible therapeutic target? J Hypertens. 2007;25(2): 265-272.

42. Papaioannou TG, Vlachopoulos CV, Alexopoulos NA, et al. The effect of heart rate on wave reflections may be determined by the level of aortic stiffness: clinical and technical implications. Am J Hypertens. 2008;21(3):334-340. 